\title{
Universal behavior of optimal paths in weighted networks with general disorder
}

\author{
Yiping Chen ${ }^{1}$, Eduardo López ${ }^{1,2}$, Shlomo Havlin ${ }^{1,3}$ and H.Eugene Stanley ${ }^{1}$ \\ ${ }^{1}$ Center for Polymer Studies, Boston University, \\ Boston, Massachusetts 02215, USA \\ ${ }^{2}$ Theoretical Division, Los Alamos National Laboratory, \\ Mail Stop B258, Los Alamos, NM 87545, USA \\ ${ }^{3}$ Minerva Center of Department of Physics, \\ Bar-Ilan University, Ramat Gan, Israel
}

\begin{abstract}
We study the statistics of the optimal path in both random and scale free networks, where weights $w$ are taken from a general distribution $P(w)$. We find that different types of disorder lead to the same universal behavior. Specifically, we find that a single parameter $\left(S \equiv A L^{-1 / \nu}\right.$ for $d$-dimensional lattices, and $S \equiv A N^{-1 / 3}$ for random networks) determines the distributions of the optimal path length, including both strong and weak disorder regimes. Here $\nu$ is the percolation connectivity exponent, and $A$ depends on the percolation threshold and $P(w)$. For $P(w)$ uniform, Poisson or Gaussian the crossover from weak to strong does not occur, and only weak disorder exists.
\end{abstract}


The study of the optimal path in disordered networks has attracted much interest in recent years, due to its relation to many complex systems of interest, including polymers 1 , 2], nanomagnet transport [3], surface growth [4], spin glasses [5], tumoral growth immune response [6], and complex networks [7]. The disorder is realized by assigning random weights $w$ to the links of the network, where the weights are drawn from a distribution $P(w)$, chosen to reflect the characteristics of the system under study. Examples include exponential disorder for tunnelling effects [3], power law disorder for driven diffusion in random media [8], lognormal distribution in conductance of quantum dots [9] and Gaussian distribution for polymers [10].

Of particular current interest is the scaling behavior of the optimal path length $\ell$ for different weight distributions [2, 7, 8]. The optimal path between two nodes is the path that minimizes the sum of the weights along the path. By definition, the weak disorder limit is obtained when almost all links contribute to the total weight of the optimal path. For this case $\ell \sim L$ [10] for a $d$-dimensional lattice of linear size $L$, and $\ell \sim \log N$ for random networks of $N$ nodes [7]. The strong disorder limit is obtained when a single link weight dominates the sum of weights along the path[11]. For this case

$$
\ell \sim L^{d_{o p t}}
$$

with $d_{\text {opt }}=1.22(d=2)$ and $d_{\text {opt }}=1.42 \quad(d=3)$ for lattices [2, 12], and $\ell \sim N^{1 / 3}$ for random networks [7]. It is commonly agreed that strong disorder arises only when $P(w)$ is broad enough.

Lacking is a general criterion to determine which form of $P(w)$ can lead to strong disorder, and a general condition when strong or weak disorder occurs. Here we derive analytically such a criterion, and support our derivation by extensive simulations. Using this criterion we show that certain power law distributions and lognormal distributions $P(w)$ can lead to strong disorder and to a weak-strong disorder crossover [12]. We also show that for $P(w)$ uniform, Poisson or Gaussian, only weak disorder occurs regardless of the broadness of $P(w)$. Importantly, we find that for all $P(w)$ that possess a strong-weak disorder crossover, the distributions $p(\ell)$ of the optimal path lengths display the same universal behavior.

A useful way to study properties of the $P(w)$ is to relate it to the uniform distribution $\pi(x)=1$, with $x \in[0,1)$. From the relation $P(w) d w=\pi(x) d x=d x, w$ can be obtained 
through the "disorder function" $f(x)$ defined as $w \equiv f(x)$, which is equivalent to

$$
x \equiv f^{-1}(w)=\int_{0}^{w} P\left(w^{\prime}\right) d w^{\prime} .
$$

In this form, every link has assigned to it a number $x$ between 0 and 1 , and its corresponding weight is given by the relation $w=f(x)$. Using $f(x)$, we can write the total weight $w_{\text {opt }} \equiv$ $\sum_{j=1}^{\ell} w_{j}$ of the optimal path as

$$
w_{\text {opt }}=f\left(x_{1}\right)+f\left(x_{2}\right)+\ldots+f\left(x_{\ell}\right),
$$

where the terms of the sum are arranged in decreasing order of values of $w_{j} \equiv f\left(x_{j}\right)$.

Next, we find the condition for strong disorder. We consider the first two terms of Eq. (3), since for strong disorder only the first term of (3) is dominant [18]. The reason for choosing only two terms is that if the second term is very small compared to first then all others can be neglected and we are in the strong disorder, while if the second term cannot be neglected all others also cannot be neglected and we are in the weak disorder. Thus only the first two terms determine the disorder. By definition $w_{2} / w_{1}=f\left(x_{2}\right) / f\left(x_{1}\right)<1$, so we can expand $f\left(x_{2}\right)$ around $x_{1}$,

$$
w_{1}+w_{2}=f\left(x_{1}\right)\left[1+\frac{f\left(x_{2}\right)}{f\left(x_{1}\right)}\right] \simeq f\left(x_{1}\right)[2-S],
$$

where

$$
\left.S \equiv \frac{d(\ln f)}{d x}\right|_{x=x_{1}}\left(x_{1}-x_{2}\right) .
$$

To see that $S$ determines the disorder strength and the weak-strong disorder crossover, we note that $(i)$ for the strong disorder limit, $w_{\text {opt }} \approx f\left(x_{1}\right)$, and therefore $f\left(x_{2}\right) / f\left(x_{1}\right) \rightarrow 0$. Since $S \simeq 1-f\left(x_{2}\right) / f\left(x_{1}\right)$, it follows that $S \rightarrow 1$, and (ii) for the weak disorder limit all weights $w_{j}$ contribute to $w_{\text {opt }}$, so $f\left(x_{1}\right) \gtrsim f\left(x_{2}\right)$, and $S \ll 1$.

To identify $x_{1}$ and $x_{2}$ in (5), we show that the strong disorder case is related to percolation [12]. The percolation problem corresponds to the construction of random networks by the removal of links with probability $1-p$. The removal of links relates to the strong disorder limit, because all links with weights $w_{i}$ larger than necessary to keep the network connected are not relevant and can be removed [13]. Therefore, the weights that are typically removed are those satisfying $w>f\left(p_{c}\right)$, where $p_{c}$ is the critical percolation threshold, the value of $p$ for which the system switches between being connected to disconnected. Hence the value of $x_{1}$ should be $p_{c}$ (in the limit of infinite system size). 
For percolation on a finite system, $p_{c}$ is not unique but follows a distribution characterized by a width which scales as $L^{-1 / \nu}$, where $\nu$ is the percolation connectivity length exponent 14 , 15]. Thus for strong disorder, both $x_{1}$ and $x_{2}$ are distributed close to $p_{c}$ with widths $L^{-1 / \nu}$. For typical values of $x_{1}$ and $x_{2}, x_{1}^{*}$ and $x_{2}^{*}$ respectively, we expect

$$
\frac{x_{1}^{*}-x_{2}^{*}}{p_{c}} \sim L^{-1 / \nu}
$$

Combining Eqs. (5) and (6), we find

$$
\left.S \sim p_{c} \frac{d(\ln f)}{d x}\right|_{x=p_{c}} L^{-1 / \nu}
$$

Using $w=f(x)$, and $f^{\prime}(x)=1 /\left.P(w)\right|_{w=f(x)}$, Eq. (17) can be expressed as

$$
S \sim \frac{p_{c} L^{-1 / \nu}}{w_{c} P\left(w_{c}\right)} \equiv A L^{-1 / \nu},
$$

where $w_{c}$ is the solution of the equation

$$
\int_{0}^{w_{c}} P(w) d w=p_{c}
$$

Equation (8) expresses $S$ as a function of the general disorder distribution $P(w)$ and $L$.

Next we extend our theory for $S$ to random networks. Percolation at criticality on Erdős-Rényi (ER) networks [16] is equivalent to percolation on a lattice at the upper critical dimension $d_{c}=6$ [7, 17]. For $d=6, L \sim N^{1 / 6}$, and $\nu=1 / 2$. Thus, using (86) we obtain,

$$
S \sim A L^{-1 / \nu}=A N^{-1 / 6 \nu}=A N^{-1 / 3}
$$

Thus, for ER networks we simply replace $L^{-1 / \nu}$ in (8) with $N^{-1 / 3}$.

Following similar arguments for a scale-free network with degree distribution $g(k) \sim k^{-\lambda}$ and $3<\lambda<4$, we can replace $L^{-1 / \nu}$ by $N^{-(\lambda-3) /(\lambda-1)}$ since $d_{c}=2(\lambda-1) /(\lambda-3)$ [17]. Thus,

$$
S \sim A L^{-1 / \nu}=A N^{-1 /\left(d_{c} \nu\right)}=A N^{-(\lambda-3) /(\lambda-1)} .
$$

For $\lambda>4$, Eq. (11) reduces to (10) 17].

Our main results, Eqs. (8), (10) and (11), suggest that since $S$ controls the disorder, for the same $S$, we expect to obtain the same optimal path behavior independent of $P(w)$.

Next we calculate $A$ for several specific weight distributions $P(w)$. We begin with the well-studied exponential disorder function $f(x)=e^{a x}$, where $x$ is a random number between 
0 and 1 [2, 3]. From Eq. (2) follows that $P(w)=1 /(a w)$, where $w \in\left[1, e^{a}\right]$. Using Eq. (9) we obtain $w_{c}=e^{a p_{c}}$, and from Eq. (18), $A$ becomes

$$
A=a p_{c}
$$

For fixed $S$, we expect to obtain the same optimal path behavior. Indeed, this has been shown to be valid [3, 18, 19].

Next we study $A$ for the disorder function $f(x)=x^{a}$, with $x$ between 0 and 1 , where $a$ is a parameter which can be positive or negative [8]. For this case the disorder distribution is a power law $P(w)=|a|^{-1} w^{1 / a-1}$. Following Eqs. (8) and (91), we obtain

$$
A=|a|
$$

Note that $a$ plays a similar role to $a$ in Eq. (12), but here $S$ is independent of $p_{c}$, which means that networks with different $p_{c}$, such as ER networks with different average degree $<k>=1 / p_{c}$, will yield the same optimal path behavior.

We further generalize the power law distribution with the disorder function $f(x)=x^{a}$ by introducing the parameter $0 \leq \Delta \leq 1$ which is defined as the lower bound of the uniformly distributed random number $x$, i.e., $1-\Delta \leq x \leq 1$. Under this condition, the distribution becomes

$$
P(w)=\frac{w^{1 / a-1}}{|a| \Delta} \quad w \in\left[(1-\Delta)^{a}, 1\right] .
$$

Again using Eqs. (8) and (9),we obtain

$$
A=\frac{\Delta a p_{c}}{\Delta p_{c}+1-\Delta}
$$

Table [ shows the results of similar analyses for the lognormal, Gaussian, uniform and exponential distributions $P(w)$. From Table — we see that for exponential function, power law and lognormal distributions, $A$ and therefore $S$ can become large. However for uniform, Gaussian and exponential distributions, $A$ is limited to a value of order 1 , so $S \ll 1$ for large $L$. Note that for these distributions, $A$ is independent of $a$ or $\sigma$. Thus only the former distributions (exponential function, power law, lognormal) are broad enough, and can lead to strong disorder behavior for the optimal path, while for the latter distributions (uniform, Gaussian and exponential), $S$ is always small and only weak disorder can appear.

To test the validity of our theory, we perform simulations of optimal paths in $2 d$ square lattices and ER networks. Random weights from different disorder functions were assigned 
to the bonds. For an $L \times L$ square lattice, we calculate the average length $\ell$ of the optimal path from one lattice edge to the opposite. For an ER network of $N$ nodes, we calculate $\ell$ between two randomly selected nodes.

Figure 1 shows our simulation results for a $2 d$ square lattice for power law and lognormal distributions. For small $L, \ell / L^{1.22}$ approachs a constant, indicating the strong disorder regime $\ell \sim L^{1.22}$, while for large $L$ it approaches to $L^{-0.22}$ indicating $\ell \sim L$. The scaled results shown in Fig.1b show collapse for the same $S$ for all distributions and therefore support Eq. (8) that $S$ controls the optimal path, and that the optimal path $\ell$ behaves in the same universal way for any broad distribution. Note that for large $S^{-\nu}$ the scaled curves approach a slope of $1-d_{o p t} \cong-0.22$ indicating the range of weak disorder.

Scaled distributions $p(\ell)$ for general disorder distributions $P(w)$ are shown in Fig. 2, in which $L, a, \Delta$ and $\sigma$ are selected to keep $S$ constant. Figure 2 suggests that for the same value of $S$, the distributions $p(\ell)$ collapse when plotted versus the scaling parameter $\ell / A^{\nu d_{o p t}}$.

Simulations for optimal paths on ER networks are shown in Fig. 3. Here we use the bombing algorithm [2, 13] to determine the path length $\ell_{\infty}$ in the strong disorder limit, which is related to $N$ by $\ell_{\infty} \sim N^{\nu_{o p t}}=N^{1 / 3}[7,20]$. We see that for all disorder distributions studied, $\ell$ scales in the same universal way with $S^{-1} \equiv \ell_{\infty} / A$. For $S \ll 1, \ell / A$ is linear with $\log \left(\ell_{\infty} / A\right)$ as expected (Fig. 3a). For large $S=A / \ell_{\infty}$ (Fig. 3b), $\ell \propto \ell_{\infty} \sim N^{1 / 3}$, which is the strong disorder behavior [7]. Thus, we see that when $N$ increases, a crossover from strong to weak disorder occurs in the scaled optimal paths $\ell / A$ vs. $S^{-1}$. Again, the collapse of all curves for different disorder distributions for ER networks supports the general condition of Eq. (8) .

Next we use Eq. (8) to analyze the other types of disorder given in Table \t that do not have strong disorder behavior. For a uniform distribution, $P(w)=1 / a$ and we obtain $A=1$. The parameter $a$ cancels, so $S=L^{-1 / \nu}$ for lattices, and $S=N^{-1 / 3}$ for ER networks. Hence for any value of $a, S \ll 1$, and strong disorder behavior cannot occur for a uniform distribution.

Next we analyze the Gaussian distribution. We assume that all the weights $w_{j}$ are positive and thus we consider only the positive regime of the distribution. We obtain $A$ as given in Table I. The disorder is controlled solely by $p_{c}$ which is related only to the type of network, and $A$ cannot obtain large values. Thus, also for the Gaussian $P(w)$, all optimal paths are in the weak disorder regime. 
Similar considerations lead to the same conclusion for the exponential distribution. Simulation results for the Gaussian distribution shown in Fig. 4 display only weak disorder (i.e. no weak-strong disorder crossover), thus supporting the above conclusions.

In summary, we find a criterion for the disorder strength $S$ on the optimal path in weighted networks for general distributions $P(w)$. We find an analytical expression, Eq. (8), which fully characterizes the behavior of the optimal path. We simulated several distributions and the results support our analytical prediction. It is plausible that the criterion of Eq. (8) is valid also for other properties in weighted networks - such as conductivity and flow in random resistor networks - due to a recently-found close relation between the optimal path and flow [3, 19].

Our results also suggest the conjecture that disorder distributions fall into two classes: those $P(w)$ that can lead to strong disorder and those that cannot. Our studies suggest that the ratio $\sigma / \mu$ determines the class, where $\sigma^{2}$ is the variance of $P(w)$ and $\mu$ the mean value. If $\sigma / \mu$ can become large $(\gg 1)$, strong disorder can occur, but if $\sigma / \mu \approx 1$ only weak disorder occurs. This criterion is valid for all the cases simulated and we conjecture that it is valid for any weighted distribution.

We thank ONR, European NEST project DYSONET, and Israel Science Foundation for support.

[1] M.D. Rintoul, J. Moon, and H. Nakanishi, Phys. Rev. E 49, 2790 (1994).

[2] M. Cieplak, A. Maritan, and J.R. Banavar, Phys. Rev. Lett. 72, 2320 (1994).

[3] Y. M. Strelniker et al., Phys. Rev. E 69, 065105(R) (2004).

[4] A. -L. Barabási and H. E. Stanley, Fractal Concepts in Surface Growth (Cambridge, NY, 1995).

[5] M. Mezard, G. Parisi, M.A. Virasoo, Spin Glass Theory and Beyond (World Scientific Pub, 1987).

[6] A. Brú, S. Albertos, J.A.López García-Asenjo, and I. Brú, Phys. Rev. Lett. 92, 238101 (2004).

[7] L.A. Braunstein et al. Phys. Rev. Lett. 91, 168701 (2003).

[8] A. Hansen and J. Kertész, Phys. Rev. Lett. 93, 040601 (2004).

[9] E. McCann and I.V. Lerner, J. Phys. Cond. Matt. 8, 6719 (1996). 
[10] I. Smailer et al., Phys. Rev. E 47, 262 (1993)

[11] This criterion can be regarded as the definition of strong disorder, see also [2].

[12] M. Porto et al., Phys. Rev. E 60, R2448 (1999).

[13] This is actually the bombing algorithm that determines the optimal path in strong disorder 2].

[14] D. Stauffer and A. Aharony, Introduction to Percolation Theory (Taylor \& Francis, London, 1994).

[15] A. Bunde and S. Havlin, Fractals and Disordered System (Springer, Berlin, 1991).

[16] P. Erdős and A. Rényi, Publ. Math. (Debrecen) 6, 290 (1959).

[17] R. Cohen et al., Phys. Rev. E 66, 036113 (2002).

[18] E. Perlsman and S. Havlin, Eur. Phys. J. B 43517 (2005).

[19] Z. Wu et al., Phys. Rev. E 71, 045101(R) (2005).

[20] S. Sreenivasan et al., Phys. Rev. E 70, 046133 (2004). 
TABLE I: Parameters controlling the optimal paths on networks for various distributions of disorder.

\begin{tabular}{|c|c|c|c|}
\hline Name & Function & Distribution & $A$ \\
\hline Inverse & $e^{a x}$ & $\frac{1}{a w} w \in\left[1, e^{a}\right)$ & $a p_{c}$ \\
\hline Power Law & $x^{a}$ & $\frac{w^{1 / a-1}}{|a| \Delta}$ & $\frac{\Delta a p_{c}}{\Delta p_{c}+1-\Delta}$ \\
\hline Lognormal & $e^{\sqrt{2} \sigma \operatorname{erf}^{-1}(2 x-1)}$ & $\frac{e^{-(\ln w)^{2} / 2 \sigma^{2}}}{w \sigma \sqrt{2 \pi}}$ & $\frac{\sqrt{2 \pi} p_{c} \sigma}{e^{-\left[\operatorname{erf}^{-1}\left(2 p_{c}-1\right)\right]^{2}}}$ \\
\hline Uniform & $a x$ & $1 / a$ & 1 \\
\hline Gaussian & $\sqrt{2} \sigma \operatorname{erf}^{-1}(x)$ & $\frac{2 e^{-w^{2} /\left(2 \sigma^{2}\right)}}{\sigma \sqrt{2 \pi}}$ & $\frac{\sqrt{\pi} p_{c} e^{\left[\operatorname{erf}^{-1}\left(p_{c}\right)\right]^{2}}}{2 \operatorname{erf}^{-1}\left(p_{c}\right)}$ \\
\hline Exponential & $-\frac{\ln (1-x)}{\zeta}$ & $\zeta e^{-\zeta w}$ & $\frac{p_{c}}{\left(p_{c}-1\right) \ln \left(1-p_{c}\right)}$ \\
\hline
\end{tabular}



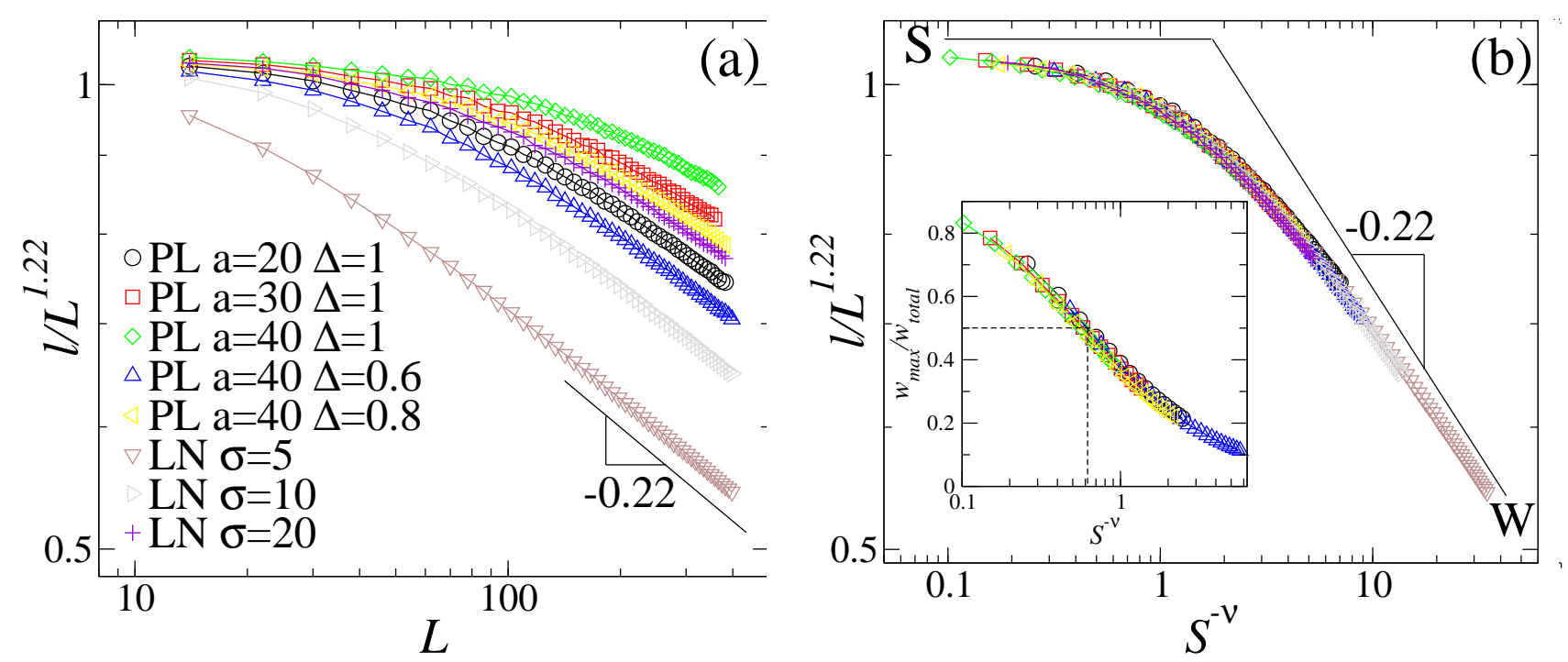

FIG. 1: Simulation results for different disorder distributions in $2 d$ lattice $(a)$ before and $(b)$ after scaling. A lower limit of $L=15$ is set to avoid the finite size effects. (a) Curves for power law disorder chosen from $x^{a}, 0 \leq x<1$, with $a$ from 20 to 40 , fixing $a=40$ and $\Delta=0.6$ or 0.8 , and lognormal distribution with $\sigma$ from 5 to 20. (b) Same results plotted in a scaled form. Two lines are drawn in $(b)$, with slopes 0 and -0.22 , indicating the theoretical behavior of optimal path in the strong and weak disorder limits respectively. The inset in (b) shows the ratio between the highest weight and total weight versus $S^{-\nu}$. A line of $w_{\max } / w_{\text {total }}=0.5$ indicates the point $S^{*}$ above which the highest weight dominates the total weight. 


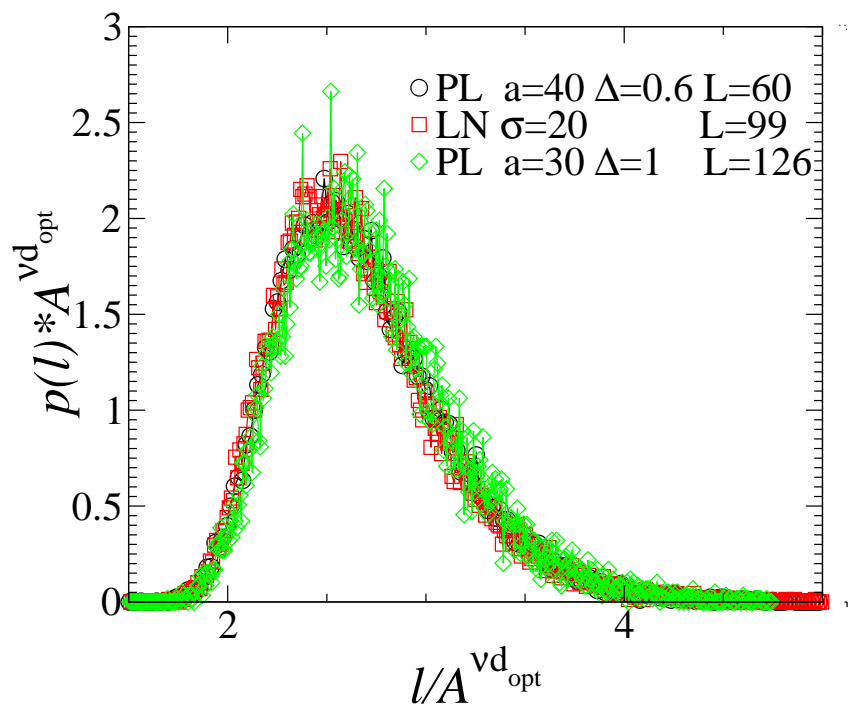

FIG. 2: Scaled distributions of $p(\ell)$ in $2 d$ lattice. Distributions used here are lognormal distribution with $\sigma=20$, power law distribution with $a=30$ and power law distribution with $a=40, \Delta=0.6$. The linear size $L$ of lattice is selected by fixing $S=0.8$. 

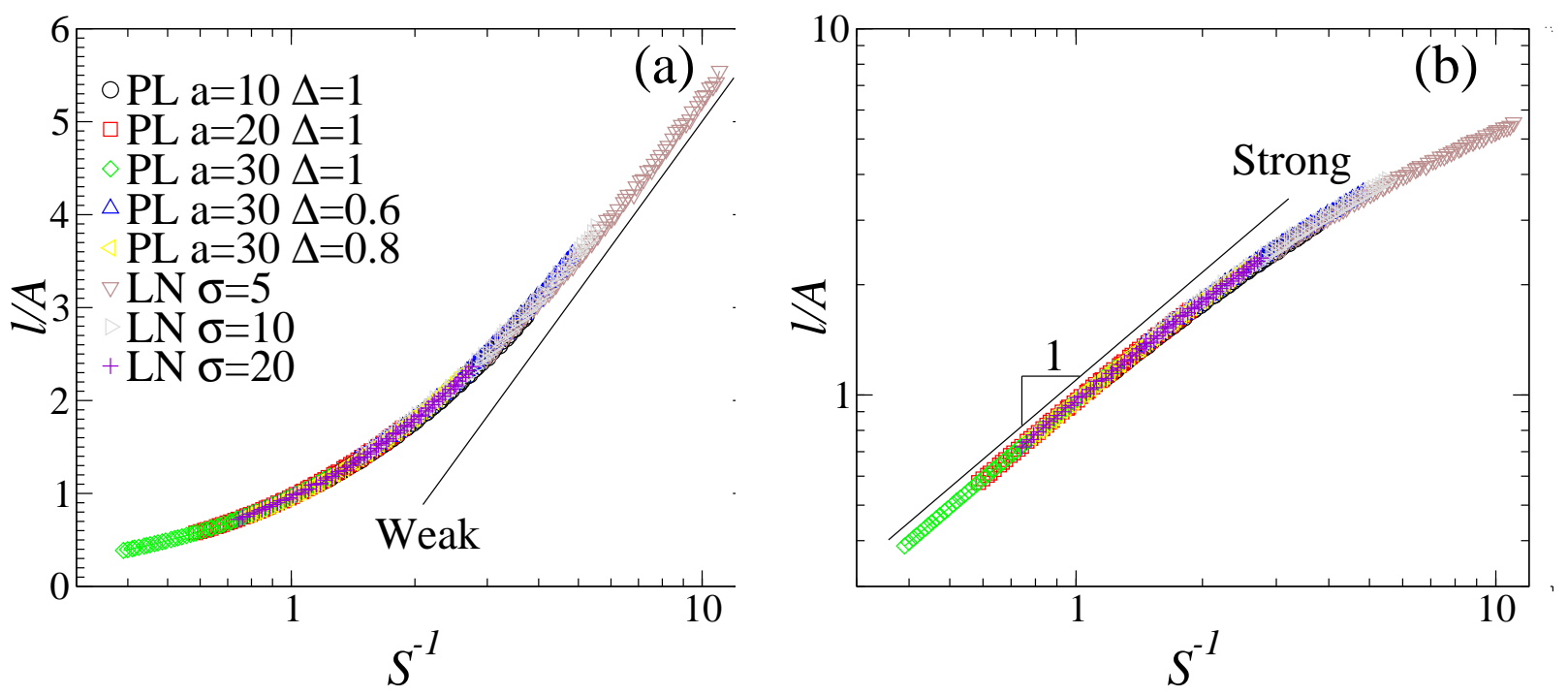

FIG. 3: The function $\ell / A$ for ER networks after scaling, where $(a)$ is a linear-log plot and $(b)$ is a log-log plot. Distributions used are power law $x^{a}$ with $10 \leq a \leq 30$ where $0 \leq x<1, x^{a}$ with $a=30$ and the range of $\Delta<x \leq 1$ with $\Delta=0.6$ or 0.8 , and lognormal distribution with $10 \leq \sigma \leq 30$. The straight line in $(a)$ indicates weak disorder and the straight line in $(b)$ indicates strong disorder. 

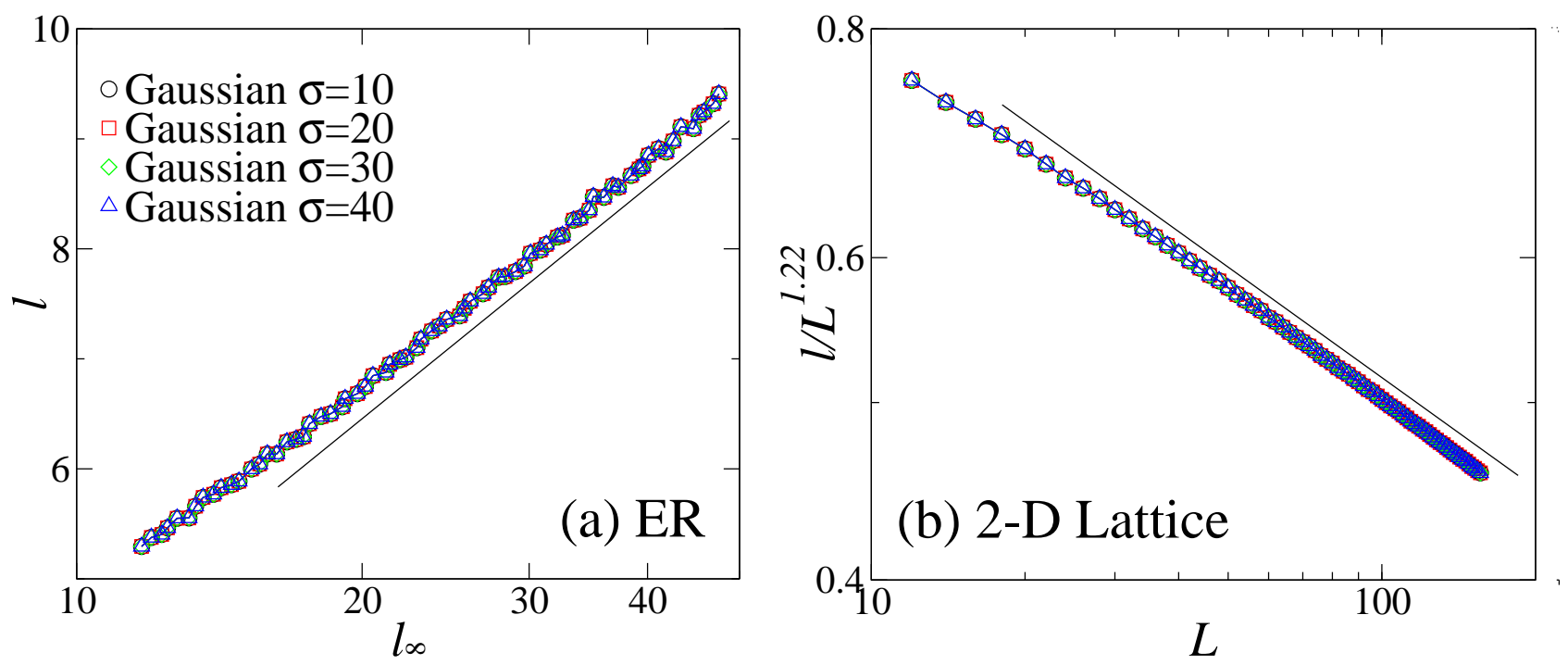

FIG. 4: The optimal path for Gaussian distribution of weights. (a) Gaussian distribution for ER networks and (b) Gaussian distribution for $2 d$ lattices. Note that these curves would collapse after scaling to the curves in figure $1 \mathrm{~b}$ in the weak disorder tail of large $S^{-\nu}$. 